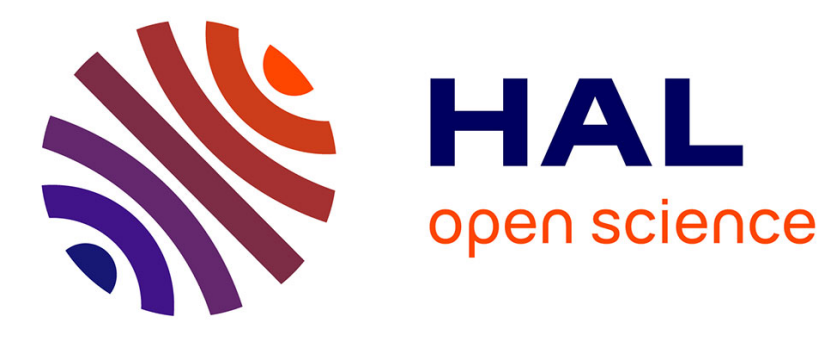

\title{
Cross-layer Monitoring in IPTV Networks
}

George Gardikis, Lemonia Boula, Georgios Xilouris, Anastasios Kourtis, Evangelos Pallis, Mamadou Sidibé, Daniel Negru

\section{To cite this version:}

George Gardikis, Lemonia Boula, Georgios Xilouris, Anastasios Kourtis, Evangelos Pallis, et al.. Crosslayer Monitoring in IPTV Networks. IEEE Communications Magazine, 2012, 50 (7), pp.76 - 84. 10.1109/MCOM.2012.6231283 . hal-00998843

\section{HAL Id: hal-00998843 https://hal.science/hal-00998843}

Submitted on 3 Jun 2014

HAL is a multi-disciplinary open access archive for the deposit and dissemination of scientific research documents, whether they are published or not. The documents may come from teaching and research institutions in France or abroad, or from public or private research centers.
L'archive ouverte pluridisciplinaire HAL, est destinée au dépôt et à la diffusion de documents scientifiques de niveau recherche, publiés ou non, émanant des établissements d'enseignement et de recherche français ou étrangers, des laboratoires publics ou privés. 


\title{
Cross-Layer Monitoring in IPTV Networks
}

\author{
Georgios Gardikis, Lemonia Boula, George Xilouris, and Anastasios Kourtis, \\ National Centre for Scientific Research "Demokritos" \\ Evangelos Pallis, Technological Educational Institute of Crete \\ Mamadou Sidibé, VIOTECH Communications
}

Daniel Négru, CNRS-LaBRI lab, University of Bordeaux I

\begin{abstract}
In a time when media content, including usergenerated, is flooding the Internet, and WebTV services are becoming more and more attractive and competitive, "fenced wall" IPTV operators need to come up with clear benefits, focusing on high content quality and guaranteed QoS/QoE. Quality guarantees can be achieved only via an end-to-end network and service management architecture, supported by an efficient monitoring system. This article discusses cross-layer IPTV service and network monitoring approaches, presenting overall aims and challenges, metrics to be monitored, and measurement strategies according to point of observation (in-network or client-side monitoring). Finally, it presents the monitoring system developed within the ALICANTE research project as an example of a complete end-to-end cross-layer monitoring framework for media services.
\end{abstract}

\section{INTRODUCTION}

As a service, IPTV can be defined as the delivery of multimedia content (mostly audio/video and associated interactive applications) over IPbased networks in a managed manner so that the service quality is monitored and ensured.

IPTV services are rapidly rolling out worldwide, with estimated global revenues increasing from $\$ 12$ billion in 2009 to $\$ 38$ billion in 2013 [1]. This growth has been strengthened by the advances in access technologies and speeds (digital subscriber line, $\mathrm{xDSL} /$ fiber to the $\mathrm{x}, \mathrm{FTTx}$ ), encoding algorithms (advanced video coding, AVC), and also in content quality (high definition, $\mathrm{HD} / 3 \mathrm{D})$.

In addition to providing profitable and attractive business cases, IPTV deployment also involves collaboration among different stakeholders. While in legacy broadcast TV systems the content, service, and network are usually owned by a single actor, in IPTV these roles are often decoupled; the content, the service, and the network may be provided by different business entities. This decoupling offers more flexibility in service deployment, but requires stringent contracts and service level agreements (SLAs) to be established among the different players. The honoring of these SLAs is critical for the overall viability of the IPTV service which is tightly associated with service assurance and provisioning of end-to-end quality of service (QoS).

Service assurance becomes imperative in a time when best effort Internet media is gaining ground; the content available on the web is rising exponentially and more users are tending to use the Internet for unmanaged media consumption. Confronting this reality, IPTV providers must provide a clear benefit in order to continue to attract subscribers into their "fenced" networks. And this clear benefit is the offering of high-quality content, presented in HD and/or $3 \mathrm{D}$, with guaranteed quality of experience (QoE). In order to achieve this, an integrated service and network management architecture is essential, supported by a real-time monitoring system. Cross-layer monitoring, employing procedures spanning from the physical to the application layer and crossing all system segments (i.e., service provider, network provider, and customer domains) is crucial for service quality assurance, fault detection, and system optimization.

In this context, this article attempts an overall approach to the subject by:

- Identifying the aims of an IPTV monitoring framework and the associated challenges

- Overviewing the quantitative monitoring metrics

- Discussing in-network and client-side measurement approaches for the collection of the aforementioned metrics

Last, it presents, as an example, an end-toend cross-layer monitoring approach for managed multimedia services, as engineered in the frame of the EU-funded ALICANTE project. 


\section{Aims AND Challenges}

A managed IPTV network is hierarchically structured. A typical structure is shown in Fig. 1, while the actual architecture varies across implementations. The super head-end is the source of nation- or continent-wide content. It feeds several local/regional video head-ends, where local content is injected. The IPTV flows are transported through one or more core/edge networks until they reach the access multiplexer, which usually uses xDSL, cable, or optical technology. At the user's premises, the customer gateway distributes the IPTV services within the home network.

Across this hierarchical delivery chain, service assurance is of key importance for the long-term viability of an IPTV provider. Indeed, television services have been traditionally associated with high quality and availability; an outage, even short, which could be common in an Internet access platform or a cellular phone network, is not at all tolerable in a television service. Even small impairments in IPTV traffic can cause severe distortion to both video and audio, which is quite annoying. That is why any issue arising at any point of the IPTV delivery chain must be promptly detected and instantly mitigated, either manually or automatically. International Telecommunication Union - Telecommunication Standards Sector (ITU-T) G.1081 [2] indicates that all domains within the provision chain, as indicated in Fig. 1, spanning from the content server up to the user's presentation devices, should be potentially under surveillance in order to be able to translate the measured data from the monitoring points into actionable knowledge.

In this context, the required functionalities of an IPTV monitoring system can be identified in two main categories: reactive (referring to the response of the monitoring system to an abnormal situation) and proactive (referring to the behavior of the monitoring system under normal operation). Whether each of the functionalities listed below is essential for a provider or not depends on the structure and scale of the IPTV platform and also the operational requirements of the provider, associated with several technical and business constraints.

Reactive functionalities ensure prompt fault identification and mitigation. These include:

-Detection of service outage or quality deterioration. IPTV services, especially multicast ones, are quite sensitive to network-level impairments. Packet losses even on the order of 0.1 percent can cause perceptible distortions to both video and audio.

- Estimation of the magnitude of the problem. A service interruption is most annoying and must be promptly recovered. But even small losses or increased jitter may degrade the quality as perceived by the user, a situation that in the long term can lead to customer attrition.

-Localization of the failure point and determination of the impact. Due to the hierarchical structure of the IPTV network, the impact of the problem strongly depends on the point of failure across the chain (Fig. 1). While a problem in the access multiplexer will affect only the viewers connected to it (normally a few tens or hun-

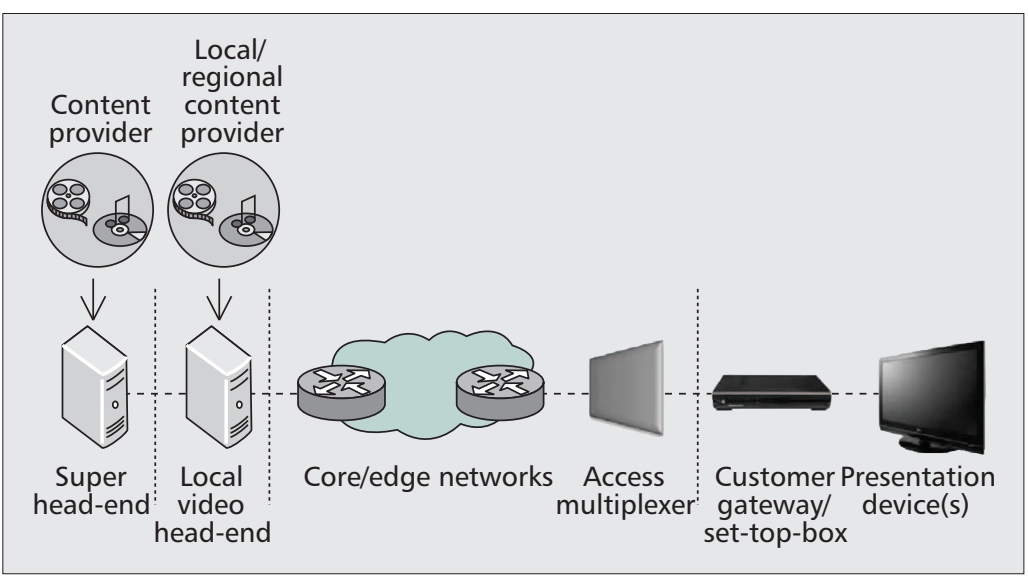

Figure 1. Typical architecture of an IPTV network.

dreds), a critical issue in the super head-end will have an impact on all customers.

-Assessment of the impact on viewer QoE. While the monitoring system will usually report an issue by means of a network- or session-level event, such as increased packet loss or jitter, it is very important to map this event to its actual impact on viewer perceived quality. This impact can depend on a great variety of factors, including codec, transport protocols, and even content type/genre and viewer preferences.

As proactive functionalities one can mention:

- Failure/outage prevention by checking the resource utilization and the workload of system components, and identifying system bottlenecks. Certain operating thresholds should be set, the violation of which triggers an alert before an outage occurs.

- Detection of SLA status. End-to-end monitoring data can be used at any time to verify that the SLA (e.g., between a service and a network provider) is honored.

- User behavior monitoring. In order to perform long-term resource planning and optimize system operation, it is useful for a monitoring system to record the user behavior, exploiting the bidirectional nature of the IPTV platform. Excluding the collection of sensitive personal data, continuous feedback from users is crucial for the IPTV provider in order to optimize the composition and provisioning of the service; for example, assign highest priority and bandwidth to more popular channels and/or reducing channel zapping time.

Last but not least, as IPTV services are usually provided as a part of a triple- or quad-play bundle, along with voice, data and mobile, IPTV monitoring should be integrateable with the monitoring systems of the other service components, so that the service provider can have a complete picture of the status of the entire bundle.

In the attempt to fulfill the aforementioned requirements, IPTV monitoring presents numerous challenges, most of which are associated with the procedure of jointly monitoring service and network status for multimedia streams that are distributed to potentially millions of users, over one or more underlying network operators.

A main obvious challenge is scalability; the 
Video Mean Opinion Score (V/MOS)

Audio Mean Opinion Score (A/MOS)

Overall Mean Opinion Score (MOS)

Peak Signal-to-noise ratio (PSNR)

Estimated PSNR (EPSNR)

Visible/Audible error rate

Lip synch drift

Channel zapping time

VoD control delay (trick latency)

Set-top-box (STB) status
A 1-5 score that estimates the perceived quality of the presented video, as assessed by the user

A 1-5 score that estimates the perceived quality of the presented audio, as assessed by the user

A 1-5 score that considers the overall user experience (combination of the above)

Peak Signal to Noise Ratio (PSNR) expressed in $\mathrm{dB}$, reflecting the distortion that has occurred between the source video stream and the output video stream (reference source required)

Estimated Peak Signal to Noise Ratio (PSNR) expressed in dB. This is an estimate of the distortion that has occurred between the source video stream and the output video stream (reference source not required)

Number of visible/audible errors per time unit

Synch drift between audio and video

Delay between channel change command and presentation of the newly selected channel

Delay between the dispatch of a START/PAUSE/RESUME command in a VoD service and its actual execution

Information about the STB status (up/down, resource usage, status of link to access network, possible failures)

Table 1. User/QoE metrics.

vast number of involved network devices, especially residential gateways, can be on the order of millions, resulting in a tremendous volume of reported events. These events must be filtered and properly processed in order to determine failures that must be recovered. This process is quite critical, since events that have a small frequency count can have a significant impact on the perceived QoE.

Another challenge is the involvement of monitoring the core, edge, and access networks; while the IPTV provider can directly observe the status of its servers and head-ends, the network delivering the service may belong to another actor (i.e., one or more network operators). The latter might not always be willing to expose overall network monitoring data to the IPTV providers.

Moreover, even in the case when network metrics are available, the combination and analysis of dependencies between network- and service-level events, probably originating from different monitoring points, in order to define the actual cause of the problem also can be quite challenging.

In the IPTV market, monitoring solutions are most often provided by the vendor of the IPTV platform itself. Again, due to service/network decoupling, it is highly likely that different management systems are used for the core/access network and for the video servers and head-ends; in this case, interoperability is an issue. Other manufacturers have introduced stand-alone monitoring platforms that are installed into an existing network in the form of distributed probes. In any case, the cost of efficiently monitoring IPTV can be quite high; but again, as explained, the cost of poor monitoring, in terms of revenue loss, eventually can be even higher.

\section{MONITORING MetRICS}

Toward fulfilling the requirements mentioned in the previous section, this section presents an overview of the metrics, that is, the quantifiable and measurable parameters that can be monitored in an IPTV system. These metrics can be categorized according to the architectural layer (i.e., application, network, transport) to which they correspond. The simultaneous collection of metrics not only from multiple points in the network, but also corresponding to multiple layers, is referred to as cross-layer monitoring. Usually, in a large-scale IPTV network, surveillance can extend down to the network layer. The extension of cross-layer monitoring to lower layers (data link and physical) is not so usual in actual largescale deployments and is more common in theoretical studies in the literature or in closed access networks, mostly wireless ones. This, for example, is the case for the cross-layer monitoring system developed in the EU-funded ADAMANTIUM project (FP7/ICT-214751), which performs IPTV and voice service optimization in third-generation $(3 \mathrm{G})$ networks based on joint surveillance at the application, network, and data link/physical layers [3]. In the present survey, data link and physical-layer metrics, such as bit error rate (BER), modulation error ratio (MER), and frame error rate (FER), which are most commonly examined in broadcasting, wireless, and cellular systems are not included.

In this context, the parameters usually monitored in an IPTV network can be categorized into:

- User/QoE metrics, which refer to the user perception and give immediate visibility of the impact of a wide range of impairments on user 
perceived quality. It must be noted here that the term QoE generally refers to the overall acceptability of an application or service, as perceived subjectively by the end user. This acceptability depends on a wide range of parameters, original content quality, encoding quality, even subscription cost for a specific service (value for money), which are not expected to change over time and whose impact can only be evaluated subjectively i.e. by a panel of test viewers. ITU-T G.1080 [4] presents a thorough presentation of QoE requirements for IPTV. A monitoring system only focuses on the estimated degradation of the QoE caused by network/service/system impairments such as delays and errors. This degradation, expressed by a drop in the mean opinion score (MOS) is calculated via algorithms that map objective parameters - commonly collected at application or network/transport level, such as video frame loss or packet loss - to the actual impact to the perceived quality.

-Application (stream) metrics are measured during the decoding and presentation process of the encoded audiovisual stream. They are commonly measured by the stream decoder, either at the client set-top box (STB) or at probe decoders distributed within the network.

- Transport/network metrics provide key information on performance of transport and network protocols (IP, UDP, RTP, etc), which can directly affect service quality. They are measured either at the client STB (edge measurement) or at several points within the network such as routers and access multiplexers (in-network measurement). These values can be used to extract the Media Delivery Index (MDI, according to RFC 4445), which is quality indicator based solely on network metrics.

Since, to the authors' knowledge, in the literature only partial lists of IPTV metrics exist, as categorized above, Tables 1-3 present an effort to aggregate the most common metrics referred to in research articles and standards/recommendations. Each metric is followed by a brief description, except in self-evident metric names. A very essential subset of these metrics, along with a reference model for IP video monitoring, mostly on end-to-end basis, can be found in ITU-T Recommendation J.241 [5].

While most of the metrics in Tables 1-3 are protocol-independent, Table 4 summarizes the most common transport-layer metrics specific to the MPEG-2 Transport Stream (TS), as defined in ETSI TR101 290 [6]. They were included since the MPEG-2 TS is the most common stream container not only in digital broadcasting systems but also in IPTV networks.

There are also other protocol-specific metrics, such as forward error correction (FEC) metrics, where FEC mechanisms are used for detection and correction of erroneous/missing packets at the client, and Reliable User Datagram Protocol (RUDP) metrics, where the RUDP protocol is employed for retransmission of UDP datagrams.

Last but not least, there are also metrics related to user behavior [7] such as user session characteristics (when people watch TV and what their attention span is across genre), channel popularity, and dynamics (how user interests are
Metric

\section{Description}

\begin{tabular}{|l|l|}
\hline Audio/video/total mean bit rate & $\begin{array}{l}\text { Average audio/video/total bitrate } \\
\text { (excluding IP overhead, FEC and } \\
\text { retransmissions) }\end{array}$ \\
\hline Audio/video/total peak bit rate & $\begin{array}{l}\text { Peak audio/video/total bitrate } \\
\text { (excluding IP overhead, FEC and } \\
\text { retransmissions) }\end{array}$ \\
\hline $\begin{array}{l}\text { Percentage of I, P, and B frames } \\
\text { impaired (by loss/discard) }\end{array}$ & \\
\hline No. of I, P, B frame packets received & \\
\hline No. of I, P, B frame packets lost & \\
\hline No. of I, P, B frame packets discarded & \\
\hline No. of buffer overflow events & \\
\hline No. of buffer underflow events
\end{tabular}

Table 2. Application (stream) metrics.

spread across channels over time) and geographical locality (whether users in the same region or DSL access multiplexer, DSLAM, show similar viewing patterns).

With regard to the observation point (i.e., where the aforementioned cross-layer metrics are measured), the monitoring procedure may take place either:

- Within the distribution network (in-network monitoring)

- At the client's premises (client-side monitoring)

The following sections present these two approaches.

\section{IN-NETWORK MONITORING}

In-network IPTV monitoring is performed within the distribution network, core, edge, and access, including the access multiplexer. It must be distinguished from the overall network monitoring performed by the network provider, since the object of surveillance is not the network itself, but the specific services which are conveyed i.e. the individual stream flows and/or the traffic aggregate to which the flows belong.

There is also a possibility that IPTV services are transported over virtualized network topologies (virtual path, virtual tree, etc.), provisioned over one or more underlying physical infrastructures. This is the case with the ALICANTE architecture, as described in the sections following. In this scenario, network measurements are performed on the provisioned virtual networks and not directly on the underlying links.

In-network monitoring mainly collects transport/network metrics, such as packet loss and interarrival jitter. These metrics are measured by 
Uncorrected packet loss ratio

Corrected packet loss ratio

Mean consecutive loss period

Max consecutive loss period

Packet loss burstiness

Packet discard ratio

Out-of-sequence packet ratio

Duplicate packet ratio

One-way delay (avg/max)

Round trip delay (avg/max)

Mean absolute packet delay variation

Mean packet-to-packet delay variation

Positive/negative jitter threshold

Positive/negative jitter percentile

Network capacity/utilization

Join/leave IGMP delay
Percentage of IP packets lost in the network

Packet loss rate after correction by Forward Error Correction or retransmission

Average length of consecutive loss periods

Maximum length of consecutive loss periods

Ratio of number of packets lost over number of packet loss events

Percentage of packets discarded due to late arrival or other reasons
Mean variation of the packet one-way end-to-end delay

Mean variation of packet interarrival time

Percentage of packets arriving within positive/negative jitter threshold

Overall capacity and utilization percentage of a certain network link or a virtual path within a network

Delay between the dispatch of the IGMP request and the finalization of the join/leave procedure in a multicast stream

Table 3. Transport/network metrics.

either the network elements themselves or monitoring devices that capture and analyze a portion of the forwarded traffic. The latter are usually connected to "mirror ports" of the routers or switches, record a portion of the overall traffic, isolate the IPTV streams, and perform measurements on the captured trace. Since recording the overall IPTV traffic can produce quite huge traces, packet filtering and sampling is often used. However, the configuration of the sampling procedure can be quite tricky, since due to the high sensitivity of media services to network losses, even small impairments - such as packet losses down to $10^{-4}$ or even lower - must be promptly detected and reported. Thus, sparse sampling must be generally avoided.

The retrieval of measured metrics is usually performed by a centralized network manager, using protocols such as Simple Network Management Protocol (SNMP). The IP Flow Information Export (IPFIX, RFC 5101) protocol can also be used for the provision of metrics related to specific IP flows. As an alternative, many modern routers and switches feature a web service interface for monitoring, which can also be employed.

In addition to transport/network metrics, innetwork monitoring may also involve higherlayer (e.g., application/stream and probably user/QoE) metrics. Since these cannot be moni- tored by the network elements, for their evaluation a set of probe modules is deployed within the network. These probes record the IPTV streams at in-network links, and process/decode them in order to assess the impact of the network on the media service in a higher-layer aspect (e.g., evaluating the video frame loss or the service MOS). It is self-evident that as the number of probes within the network increases, it is easier to detect and locate an issue which could degrade service quality. However, in this case, not only the cost of probe deployment, but also the complexity of collecting and jointly assessing a large set of measurements, must be taken into consideration.

\section{CLIENT-Side Monitoring}

Client-side monitoring is performed at the customer premises, at three different points: at the customer network gateway, at the decoder/STB and at the presentation device (i.e. after stream decoding).

The customer gateway can collect transport/network metrics such as packet loss and interarrival jitter from the real-time analysis of the incoming network flows. Application/ stream parameters such as I/P/B frame loss and MPEG-TS-specific metrics are monitored during the stream demultiplexing and decoding proce- 


\begin{tabular}{|l|l|}
\hline Metric & Description \\
\hline PCR Jitter & Average program clock reference (PCR) jitter level \\
\hline TS Sync loss & Loss of synchronization at MPEG transport layer \\
\hline Sync byte error & Count of invalid MPEG transport sync bytes (i.e., not 0x47) \\
\hline Continuity count error & Count of transport packets in incorrect order, duplicate packet or lost packet \\
\hline Transport error & Count of transport packets with transport error indicator in MPEG transport header set \\
\hline PCR error & Count of discontinuities in PCR \\
\hline PCR repetition error & Time interval between two successive PCR values more than 40ms (event count) \\
\hline PCR discontinuity indicator error & Difference between two consecutive PCR values is over 100 ms without discontinuity bit set (event count) \\
\hline PTS error & Interval between presentation time stamps (PTSs) more than 700 ms (event count) \\
\hline
\end{tabular}

Table 4. MPEG-2 transport stream metrics (as defined in ETSI TR 101 290).

dure at the decoding STB, and can be directly reported. User behavior metrics such as channel popularity are also monitored here.

A challenging issue in client-side monitoring is the derivation of user/QoE metrics, especially the video/audio quality expressed by the MOS. In the simplest approach, the MOS is directly calculated from network and application metrics using psychometric models. The latter also take into account stream parameters, such as bit rate, resolution, and image complexity, and try to map the impairments introduced by the network to their actual impact on the perceived quality of the service (i.e., estimate how much the objective picture or sound quality is degraded). Numerous algorithms are present in the literature that follow this approach. In this category, the "V-factor" metric [8] is probably the most popular in the industry. In any case, the error concealment capabilities of the decoder must be taken into account, which vary across different devices. An error loss pattern that produces a very annoying result in a certain decoder may be quite efficiently concealed by another model and have a significantly lower impact on service quality.

A more complicated approach, which can include the error concealment stage, is the direct analysis of the decoded image or sound. Imagebased quality assessment is a computationally intensive procedure, which can, however, yield results quite close to user perception. Full-reference (FR) metrics, such as the peak signal-tonoise ratio (PSNR), need both the original and received/distorted images, and their applicability in large-scale IPTV networks is restricted. Noreference (NR) mechanisms analyze only the received signal in an effort to determine the introduced distortion, usually in the form of blockiness. Reduced-reference (RR) algorithms involve processing both the original stream (at the server) and the received one (at the client), and the derivation of a very-low-bit-rate image description stream, which can be quite efficiently used to assess the overall distortion. A quite popular algorithm of this kind is the Video
Quality Metric (VQM), developed by NTIA [9] and included in ITU Recommendation J.144.

All the aforementioned cross-layer metrics at the client side may be retrieved from the corresponding devices via SNMP or web-servicesbased protocols. Again, as described in the previous section, it is also possible to deploy probes at the client network in order to perform more sophisticated and computationally intensive measurements that are not supported by the existing devices. The probes are usually connected at the gateway output (i.e., in parallel with the STB). They perform real-time network traffic analysis, and usually also proceed to stream demultiplexing and decoding in order to retrieve application- and user-layer parameters. Probes may also be connected at the STB/decoder output in order to process the analog or digital audiovisual signal and assess the picture and sound quality via the aforementioned RR or NR evaluation techniques.

\section{THE ALICANTE MONITORING APPROACH FOR MANAGED Networked Media ECOSYstems}

Combining most of the aforementioned approaches and techniques, this section presents a prototype architecture for a cross-layer monitoring system for managed media delivery platforms, as designed and developed within the EU-funded ALICANTE research project (FP7/ICT-248652) [10]. The aim of ALICANTE is to design and deploy a so-called networked media ecosystem, a composite environment involving multiple service/content providers (S/CPs), network providers (NPs), and end users (EUs), who are collaboratively engaged in the production, sharing, and communication of rich media services - including IPTV.

A fundamental element of the ALICANTE architecture is the "HomeBox" (HB), an enhanced media-centric residential gateway used not only for receiving and consuming media 


In the ALICANTE sys-
tem, content stems
from the S/CP
servers/headends,
traverses one or
more associated Vir-
tual Networks
(VCANs), reaches the
HomeBoxes of the
users who have sub-
scribed to the ser-
vice, and is finally
presented in one or
more User Terminals
behind the receiving
HomeBoxes.

In the ALICANTE sys-

em, content stems

$S / C P$

traverses one or

sociated Vir-

(VCANs), reaches the users who have subscribed to the serpresented in one or more User Terminals HomeBoxes.

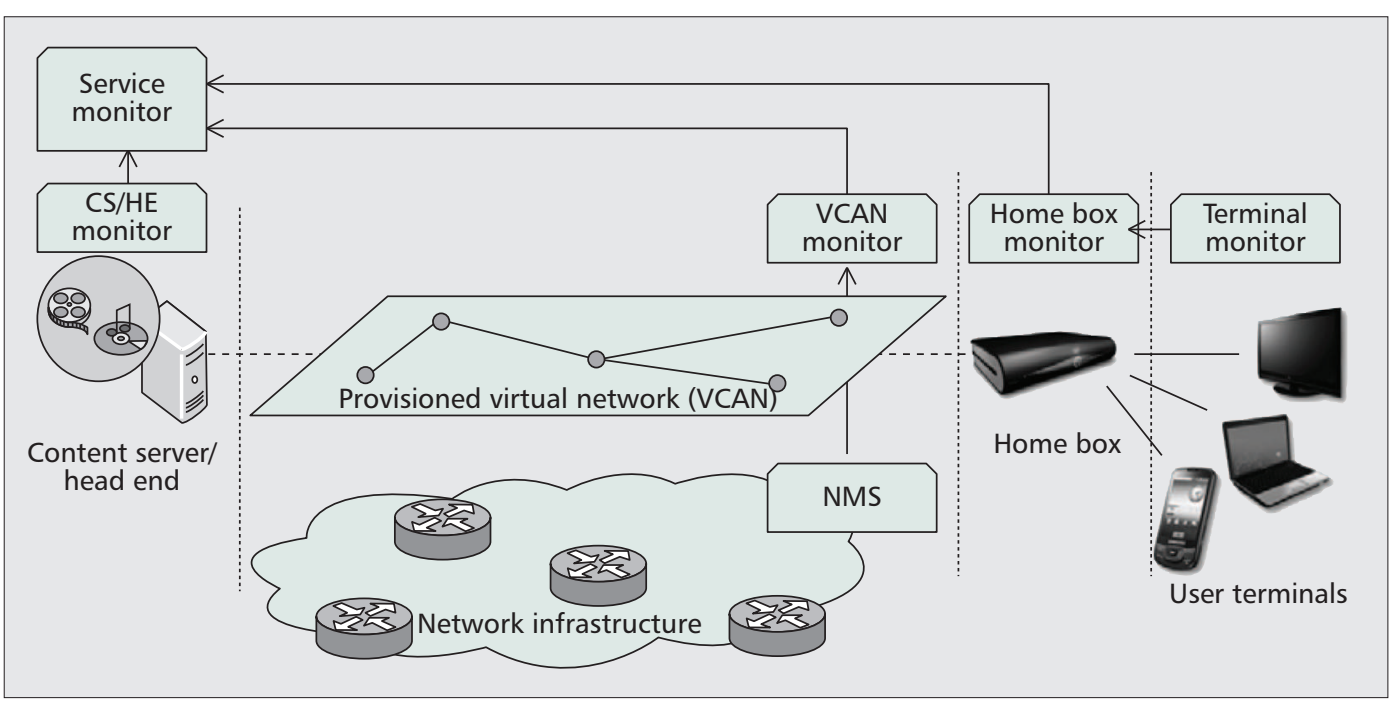

Figure 2. Architecture of the ALICANTE monitoring subsystem.

streams, but also for producing and serving usergenerated content (UGC). Multimedia services are delivered from $\mathrm{S} / \mathrm{CPs}$ to HBs or among $\mathrm{HBs}$ over dedicated virtual networks (virtual contentaware networks - VCANs in the ALICANTE terminology), deployed by one or more underlying NPs. A more in-depth presentation of the ALICANTE architecture and its features can be found in [11].

In the ALICANTE system, content stems from the S/CP servers/headends, traverses one or more associated virtual networks (VCANs), reaches the HomeBoxes of the users who have subscribed to the service, and is finally presented in one or more user terminals behind the receiving HomeBoxes.

Maximization of QoS and QoE of media services in the ALICANTE system is achieved a) via dynamic adaptation of media streams, taking place in the network and also at the edges, and b) via content-aware mechanisms in the network, performing automatic service recognition and differentiation (i.e., prioritized handling of sensitive media streams). In order to efficiently accommodate all these operations, including adaptation, real-time information from the entire media delivery chain is needed. In this context, cross-layer monitoring is an essential element in the ALICANTE concept. The proposed distributed monitoring subsystem, as described in this section and illustrated in Fig. 2, achieves thorough end-to-end cross-layer monitoring on a real-time basis. It is fully modular, relying on distributed monitoring modules and also scalable, since the extent of data collection and processing can be adjusted at any time, according to service popularity. Metric acquisition is achieved via monitoring the elements involved in service provisioning themselves, without the need to insert additional probe devices for data collection.

Service monitoring in ALICANTE spans across the S/CP servers and head-ends, the virtual networks delivering the service, and the user's HomeBoxes and terminals. At each of the four aforementioned domains, a specific set of crosslayer metrics is collected. Depending on the point of measurement, the following four sets of metrics are identified, corresponding to different architectural layers:

- Host metrics - such as host status, CPU/memory utilization, interface utilization, and number of services handled

- VCAN metrics - such as VCAN nominal and available capacity, average delay, loss, and jitter for each traffic aggregate within the VCAN

- Session metrics - such as per-session packet loss, jitter and reordering measured at transport/session layer

- Application/QoE metrics — such as video and audio MOSs

The collection of the measured metrics at each domain is undertaken by a dedicated monitoring module:

- The content server/head-end monitor monitors the status, load, and resources (host metrics) of each of the $\mathrm{CS} / \mathrm{HE}$ involved in media service provisioning, thus detecting possible malfunctions or overloads.

- The VCAN monitor collects network metrics from the network management system (NMS) of the NP, which are in turn retrieved via SNMP requests to each of the involved network elements. These values are translated into aggregate metrics for each VCAN. In this sense, the network provider does not expose the overall network performance data to the S/CP, but only the metrics related to the virtual network associated with the service.

- The HomeBox monitor measures parameters specific to the HomeBox (host metrics, corresponding to HomeBox resources) and also to the session received (session metrics) via realtime analysis of the media flows that are received.

- The terminal monitor measures parameters specific to the terminal (host metrics, corresponding to terminal resources) and to the session received (session metrics). It also estimates the perceived quality (QoE metrics, audio/video MOS) of the presented service based on session parameters and appropriate psychometric models that map traffic impairments to visual/acoustic distortion. 
All the measured data are collected from the respective monitoring modules, formatted in XML structure and communicated over SOAP interfaces. Terminal metrics are provided via the HomeBox, which in this case acts as a proxy. The service monitor aggregates all metrics and presents to the service manager a complete picture of the service provisioning chain.

However, service monitoring in ALICANTE goes beyond the standard centralized paradigm; it also provides, in a decentralized manner, an increased level of awareness on each point of the service delivery chain, thus enabling real-time cross-layer and crossdomain interactions and optimizations. This constitutes the main innovative aspect of the ALICANTE monitoring system. Monitoring modules in ALICANTE are modular independent entities, and feature open interfaces for the provision of metrics to any (authorized) function within the system. In this context, in addition to being aggregated at the service monitor, monitoring parameters are also shared, diffused across the entire ecosystem, and collaboratively exploited by all involved actors; they can be exposed to the media applications as well. This collaborative approach presents the following added-value features:

- Closer synergy between the network and service providers via the controlled exposure of VCAN metrics to the S/CP. This enables network-aware service management, that is, dynamic planning of service deployment, including real-time adaptation strategies and also admission control, according to the load and conditions of the provisioned virtual network.

-Facilitation of network-aware applications via provision of network metrics to media applications (in a way similar to the ALTO concept described in RFC 5693). This also includes "network distance" estimation for best peer or best server selection (for peer-to-peer or client-server applications, respectively).

-Facilitation of context-aware applications via provision of terminal monitoring parameters and information to media applications

-Exploitation of network monitoring information for in-network rate adaptation of media streams according to network load, achieving optimal network resource usage.

- Facilitation of client-side media adaptation, using terminal and HomeBox metrics. In this way, the HomeBox adapts the service in real time, during local redistribution, according to the capabilities and status of the associated terminals.

Validation of the ALICANTE monitoring architecture will take place in two phases. First, each one of the modules will be independently validated and evaluated in a laboratory testbed, under emulated network conditions in a variety of scenarios. The aim will be to assess the responsiveness, accuracy, and scalability of the monitoring procedures. At the second stage, the entire integrated ALICANTE system will be deployed at four large-scale pilot sites, and its operation will be assessed in "real-life" operation, involving a number of end users and use case scenarios.

\section{CONCLUSIONS}

Given the necessity for monitoring and management in IPTV networks and services, this article presented a survey of methods and approaches for IPTV monitoring. In any case, thorough monitoring can be achieved only via a cross-layer approach, spanning all domains involved in IPTV service provisioning. The presented architecture, as designed for the ALICANTE media network, not only achieves end-to-end service monitoring, but its distributed nature also promotes collaboration among actors and domains, supports the deployment of network- and context-aware services, and facilitates real-time service adaptation for improved resource usage and optimized user experience.

\section{ACKNOWLEDGEMENT}

This work is supported by the research project ALICANTE, funded by the European Commission within the Seventh Framework Programme under grant agreement no. 248652.

\section{REFERENCES}

[1] "IPTV Global Forecast - 2009 to 2013, Semiannual IPTV Global Forecast Report," Nov. 2009, MRG Group Inc.

[2] ITU-T Rec. G.1081, "Performance Monitoring Points for IPTV," Oct. 2008.

[3] L. Boula, H. Koumaras, and A. Kourtis, "An Enhanced IMS Architecture Featuring Cross-Layer Monitoring and Adaptation Mechanisms," ICAS '09), Valencia, Spain, Apr. 20-25, 2009.

[4] ITU-T Rec. G.1080, "Quality of Experience Requirements for IPTV Services," Dec. 2008.

[5] ITU-T Rec. J.241, "Quality of Service Ranking and Measurement Methods for Digital Video Services Delivered over Broadband IP Networks," Apr. 2005.

[6] ETSI TR 101290 V1.2.1, "Digital Video Broadcasting (DVB); Measurement Guidelines for DVB Systems," tech. rep., May 2001

[7] M. Cha et al., "Watching Television Over an IP Network," IMC '08, Oct. 20-22, 2008, Vouliagmeni, Greece.

[8] S. Winker and P. Mohandas, "The Evolution of Video Quality Measurement: From PSNR to Hybrid Metrics," IEEE Trans. Broadcasting, vol. 54, no. 3, Sept. 2008, pp 660-68.

[9] M. Pinson and S. Wolf, "A New Standardized Method for Objectively Measuring Video Quality," IEEE Trans. Broadcasting, vol. 50, no. 3, Sept. 2004, pp. 312-22.

[10] ALICANTE EC FP7 project (MediA Ecosystem Deployment through Ubiquitous Content-Aware Network Environments), http://www.ict-alicante.eu/

[11] G. Gardikis et al., "Media Ecosystem Deployment in a Content-Aware Future Internet Architecture," Proc. 2011 IEEE Symp. Computers and Communications, June 28-July 1 2011, Corfu, Greece, pp. 544-49.

\section{BIOGRAPHIES}

GEORGIOS GARDIKIS [M'02] received his Diploma in electrical and computer engineering from the National Technical University of Athens in 2000 and his Ph.D. from the same university in 2004. His expertise lies in the fields of digital terrestrial and satellite broadcasting, distribution networks for multimedia service provisioning, QoE assessment, and novel mechanisms for application/network coupling. Since 2004, he has been collaborating with the National Center for Scientific Research "Demokritos" and the Centre for Technological Research of Crete in the framework of national and EU-funded R\&D projects, including FP6/IST IMOSAN, in which he served as technical coordinator. He has also collaborated with the University of the Aegean, conducting a national research project regarding experimental DVB-H deployment, and with the Ministry of Transport and Communications as a consultant on digital broadcasting issues. At present, he is an associate researcher at the Institute of Informatics amd Telecommunications in the NCSR "Demokritos" and a visiting assistant
Service monitoring in

ALICANTE spans

across the $S / C P$

servers and

head-ends, the

virtual networks

delivering the service,

and the user's

HomeBoxes and

terminals. At each of

the four aforemen-

tioned domains, a

specific set of cross-

layer metrics is

collected. 
professor at the Department of Applied Informatics and Multimedia, TEI of Crete. He is a member of the IEEE Communications and Broadcast Technology Societies, the W3C Web and TV Interest Group, and the Technical Chamber of Greece since 2001 . He has more than 40 publications in international journals and refereed conferences/workshops.

LEMONIA BOULA received her Diploma as an electrical and computer engineer from the National Technical University of Athens. She worked at the Digital Telecommunications Laboratory at the Institute of Informatics and Telecommunications of NCSR "Demokritos" from 2008 to 2011 as a research associate and participated in the EU-funded ICT ALICANTE and ICT ADAMANTIUM projects. She received her M.Sc. degree on databases and information systems from the University of Athens in 2010. Her current research interests include media service provisioning, 3G network performance, and database design and management.

GEORGE XILOURIS received his physics degree in 1999 from the University of Ioannina, and his M.Sc. in automation control systems from the National Technical University of Athens in 2001. He joined National Center of Scientific Research "Demokritos" in 2001 where he currently works as an associate researcher. He has collaborated with NCSR Demokritos in several EU funded R\&D projects. Additionally he is a visiting assistant lecturer at the Department of Applied Informatics and Multimedia at TEI of Crete since 2004. His current research activities involve future multimedia delivery networks, network resource management, and network virtualization. He is a member of the IEEE Communications Society and the W3C Web and TV Interest Group. He has more than 30 publications in international journals and refereed conferences/workshops.

ANASTASIOS KOURTIS is research director and currently deputy director of the Institute of Informatics and Telecommunications of the NCSR "Demokritos." He has extensive experience in EU funded projects and he has been project manager in four of them. His technical and research activities have been in the areas of wired and wireless broadband network infrastructures, QoS, data encryption techniques, network management, and network virtualization. He has supervised a number of Ph.Ds and is the author or co-author of more than 100 scientific papers.

EVANGelos PALLIS received his B.Sc. in electronic engineering from the Technological Educational Institute of Crete in 1994, his M.Sc. in telecommunications from the University of East London in 1997, and his Ph.D. in telecommunications from the University of East London in 2002. Currently he is an associate professor at the Applied Informatics and Multimedia Department, School of Applied Technology, Technological Educational Institute of Crete. He also acts as director of the Research and Development of Telecommu- nication Systems Laboratory (PASIPHAE) of the same department. His research interests are in the fields of wire less and mobile communication systems, digital broadcasting technologies and interactive television systems, QoS/QoE techniques, and network management technologies. He is member of IEE/IET and IEEE ComSoc, and a dis tinguished member of the Union of Regional Television Broadcasters of Greece.

MAMADOU SIDIBE received his M.Sc. degree in computer science from the University of Versailles in 2003, in the fields of QoS monitoring and adaptation systems over heterogeneous networks. From 2004 to 2008 he worked as an associate researcher at the CNRS-PRISM laboratory and participated in several European collaborative projects (IST FP6 ENTHRONE 1 \& 2, ATHENA, IMOSAN). In 2008 he joined VIOTECH Communications as a research and development engineer, and has been bringing his expertise in multimedia and network monitoring into various nationa and European projects (ICT FP7 ADAMANTIUM MULTI@HOME and ANR ARDHMANN) as well. He received his Ph.D. in 2010 in the field of QoS and QoE monitoring systems at the network, application, and service levels. He is currently specializing in next generation media-aware home or residential gateways, enabling content, context and service awareness at the network level and network awareness at the service level. He is coordinating the design of a future Internet enabled media-aware home gateway and related monitoring tasks in the ICT FP7 ALICANTE project.

DANIEL NEGRU received his M.Sc. degree in computer science from the University of Pierre and Marie Curie, Paris 6, in 2002. He worked in the center of research of Motorola, Paris, specifying and developing a complete IPv6 stack from scratch, including Mobile IPv6 and multimedia components. Between 2003 and 2006, he worked at the CNRS laboratory, Paris area - a French computer science research center that does research in the underlying technologies for the next generation global information infrastructure - and participated in several national and European projects, such as ANR RIAM NMS, IST FP6 ATHENA, IST FP6 ENTHRONE, IST FP6 ENTHRONE2, and IST FP6 IMOSAN. He received his Ph.D. in 2006 in the field of broadcast and Internet convergence solutions at the network and service levels. In 2007, he became an associate professor at ENSEIRB School of Engineers/University Bordeaux, specializing in multimedia and networking. His main fields of research reside in the domains of mobility and multimedia over heterogeneous networks, IMS convergence, and service accessibility and adaptation. He is currently specializing in media-aware networks, including content, context, and service awareness at the network level and network awareness at the service level. He is coordinating the ICT FP7 ALICANTE project that tackles these fields. 